\title{
Filigrane
}

Écoutes psychothérapiques

\section{Les supervisions du cursus}

\section{Bernard Brusset}

Volume 22, numéro 1, printemps 2013

URI : https://id.erudit.org/iderudit/1017347ar

DOI : https://doi.org/10.7202/1017347ar

Aller au sommaire du numéro

\section{Éditeur(s)}

Revue Santé mentale au Québec

\section{ISSN}

1192-1412 (imprimé)

1911-4656 (numérique)

Découvrir la revue

\section{Citer cet article}

Brusset, B. (2013). Les supervisions du cursus. Filigrane, 22(1), 71-85. https://doi.org/10.7202/1017347ar

\section{Résumé de l'article}

L'auteur propose une réflexion sur les supervisions telles qu'elles trouvent place dans le cursus de formation de l'Institut psychanalytique de Paris (SPP), et, diversement, ailleurs depuis 1920 à l'Institut de Berlin. Elles aboutissent à l'habilitation ou non de l'analyste en formation lors des réunions dites de fin de cursus. L'expérience de la supervision comporte des contradictions qui lui sont inhérentes et de multiples enjeux. Elle peut être considérée comme un processus qui a des étapes différentes d'un cas à l'autre et des effets négatifs possibles. Elle a pour finalité l'appropriation de la fonction analytique dans la quête de vérité qui définit l'éthique de la psychanalyse.
Ce document est protégé par la loi sur le droit d'auteur. L'utilisation des services d'Érudit (y compris la reproduction) est assujettie à sa politique d'utilisation que vous pouvez consulter en ligne.

https://apropos.erudit.org/fr/usagers/politique-dutilisation/ 


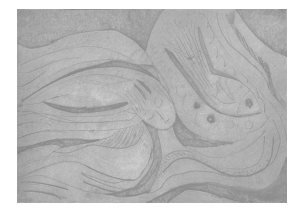

\section{Les supervisions du cursus}

\section{Bernard Brusset}

L'auteur propose une réflexion sur les supervisions telles qu'elles trouvent place dans le cursus de formation de I'Institut psychanalytique de Paris (SPP), et, diversement, ailleurs depuis 1920 à I'Institut de Berlin. Elles aboutissent à I'habilitation ou non de l'analyste en formation lors des réunions dites de fin de cursus. L'expérience de la supervision comporte des contradictions qui lui sont inhérentes et de multiples enjeux. Elle peut être considérée comme un processus qui a des étapes différentes d'un cas à l'autre et des effets négatifs possibles. Elle a pour finalité I'appropriation de la fonction analytique dans la quête de vérité qui définit l'éthique de la psychanalyse.

\section{Introduction}

ans mes fonctions de directeur de l'Institut psychanalytique de Paris (de la SPP), je poursuis une réflexion sur les pratiques de la supervision dans le cadre du cursus. Celle-ci m'a conduit à une interrogation plus générale sur la place, universelle depuis 1920, de la supervision dans la transmission de la psychanalyse et les procédures d'habilitation des psychanalystes. Je défends l'idée quelle donne lieu, au moins idéalement, à un processus de formation et plus précisément, à la transformation du psychothérapeute en psychanalyste. Cette mutation est directement en rapport avec la réélaboration à rôles inversés de l'expérience de la psychanalyse personnelle souvent encore en cours.

\section{La supervision ici et là}

Lidée que la supervision a un rôle majeur dans la transmission de la psychanalyse est très largement partagée, même par ceux qui contestent la notion de formation psychanalytique. Plusieurs formules sont utilisées dans des initiatives personnelles hors institution, notamment pour les psychothérapies: le supervisé parle de patients différents selon son choix, selon ses préoccupations, les problèmes qui se posent à lui, donc il s'agit d'abord du contretransfert. La supervision prolonge, sinon l'analyse personnelle, du moins sa perlaboration et peut, pour certains, être faite par l'analyste de l'analyse 
personnelle: une forme d'analyse secondairement didactique. Dans cette pratique, en dehors de certains groupes lacaniens, aucune institution ne vient en tiers et il n'y a de règles que celles que se donnent les protagonistes. On peut concevoir que, comme dans les groupes de médecins de Balint, le supervisé parle du dernier patient qu'il a vu ou de celui qui le préoccupe.

Beaucoup d'analystes valorisent la supervision redéfinie comme échanges interanalytiques, de préférence hors institution, dans des groupes privés dont les participants sont cooptés. L'institution, du fait de la hiérarchie, de la cléricature des formateurs, de la place faite au savoir (le sujet supposé savoir), serait une machine dé-formatrice (Viderman, 1980). La constitution de l'espace psychanalytique requiert une spontanéité créatrice par rapport à laquelle la supervision et le cursus institués ne pourraient être que normalisants et stérilisants. Dans cette optique toute institution est suspecte d'un effet d'endoctrinement. En revanche, pour d'autres, la constitution du cadre interne de l'analyste, et son contre-transfert en tant qu'il précède le transfert supposent une formation dans un cadre institutionnel réglementé. Il est considéré comme donnant fondement à l'identité professionnelle de l'analyste.

Tel est bien l'objectif quand la supervision fait partie du cursus de formation selon la formule de l'Institut de Berlin en 1920, formule conservée sans grande modification par l'IPA, exemplairement dans ce qu'il est convenu d'appeler «le modèle Eitingon». Il est diversement mis en pratique, souvent (heureusement) en excluant l'intervention de l'analyste de l'analyse personnelle dans le cursus, ce qui est la règle dans «le modèle français ». L'analyse personnelle, avec au moins trois séances par semaine, et d'une durée supérieure à trois ans, est préalable à la candidature à l'admission au cursus, et donc aux supervisions et aux séminaires de l'Institut, lesquels sont dits « validants».

\section{L'Institut psychanalytique de Paris}

D'un point de vue pratique, le choix du superviseur, outre la commodité de l'horaire et du domicile, met en œuvre des affinités électives (éventuel transfert du transfert), mais aussi la réputation et ses fluctuations (il y a des modes). La réputation d'exigence et de longue durée joue négativement.

La validation de la supervision n'est pas le fait du superviseur mais de la commission de validation du cursus : c'est un principe qui confère à l'institution comme tiers le pouvoir de déclarer le cursus clos et validé. Il évite, théoriquement, que le supervisé se sente jugé directement par son superviseur, «juge à terme» (Guillaumin, 1999). Mais la commission n'invalide 
qu'exceptionnellement une supervision contre l'avis des deux superviseurs, eux qui ont donné le feu vert pour la demande de fin de cursus. Le dispositif institutionnel a valeur symbolique et de référence commune, ce qui n'est pas indifférent.

Lors des réunions dites de fin de cursus, les superviseurs utilisent pour leur rapport les notes remises par leur supervisé et leurs propres observations. Ils donnent des exemples illustratifs du travail de supervision: des séquences d'analyse comportant des interprétations et leurs effets, l'analyse d'un rêve, etc. L'évaluation est alors l'objet d'un travail de la sous-commission dont les membres apportent des points de vue complémentaires pour comprendre l'évolution de la supervision, la progression du candidat, les transformations apparues, le processus d'appropriation de la fonction (plus ou moins réussi, il peut conduire à la demande d'une troisième supervision ou, seulement à l'initiative du candidat, à la reprise d'une analyse personnelle). Les « commissaires» responsables de l'admission au cursus sont invités à préciser ce qu'il en était initialement. La réflexion sur la pertinence des critères initiaux (parfois lointains) peut s'en trouver enrichie.

Le coordinateur, fonction instaurée il y a seulement quelques années, reçoit le candidat à la clôture de son cursus - le candidat avait préalablement choisi son coordinateur parmi trois noms proposés par le Comité de direction de l'Institut. Devant la sous-commission, le coordinateur rend compte de l'entretien de clôture et de ce qu'il appris du parcours d'ensemble de la formation du candidat, avant le cursus et au cours de celui-ci, donc de son expérience clinique, de celle des psychothérapies, des supervisions, des séminaires et des groupes de travail. De plus, l'analyste en formation est invité à donner sa propre évaluation de son cursus qui s'achève.

\section{Les réunions de fin de cursus}

Il arrive que le superviseur parle du patient comme s'il avait lui-même conduit la cure, et pas du supervisé qui semble n'avoir été qu'un moyen dont il n'y a rien à dire. La supervision est évaluée par les changements obtenus chez le patient, et elle est validée par la réussite de l'analyse. Mais le bon résultat peut dépendre davantage du patient que de l'analyste.

D’une manière générale, il est difficile de rendre compte devant la commission d'une relation privée faite de satisfaction et d'insatisfaction, de transfert (positif et négatif) et de contre-transfert (positif et négatif) dans la durée, et du fait de la complexité de la situation, de la multiplicité des niveaux impliqués, de ses enjeux narcissiques, du fait aussi que le superviseur dispose de 
beaucoup d'éléments (dont les notes aide-mémoire données par le supervisé) qu'il doit, par un travail préparatoire, réduire et schématiser. La qualité analytique du travail du supervisé ne saurait être évaluée par des critères objectifs, ce qui serait dangereux, anti-analytique, car susceptible d'impliquer un profil standard alors que les critères sont variables d'un cas à l'autre. En fait, bien qu'implicites, ils comportent généralement les mêmes repères dont l'instauration et le maintien du cadre, les modes d'intervention et d'interprétation, l'attention portée aux effets produits, la perception des transferts, l'autoanalyse du contre-transfert, le mode d'utilisation de la supervision.

Le superviseur est lui-même supervisé en quelque sorte par la commission faite de ses pairs qui sont invités à s'imaginer à sa place. D'où généralement un consensus groupal pour éviter les critiques, et souvent, de manière sans doute regrettable, d'éviter de faire état des différences dans la conception même de la supervision et partant, de la pratique analytique elle-même (un aspect particulier : accepte-t-on ou non la psychothérapie antérieure à l'analyse pour le cas de supervision, ou seulement les deux entretiens préliminaires que préconisait Bouvet; est-ce encore possible?).

Les difficultés de l'évaluation, par-delà l'enjeu de reconnaissance institutionnelle, ne sont pas sans rapport avec la complexité de la situation de supervision elle-même.

\section{La situation de supervision}

Elle a des parentés avec la situation analysante qu'elle redouble: le supervisé rapporte ce qu'il a entendu et ce qu'il n'a pas entendu, ce qui lui a échappé et ce qui lui échappe encore, à quoi il va être confronté : l'inconscient dont il est alors le dupe. Cela le met lui-même en situation analytique, dans les limites éventuelles de son analyse personnelle. Le superviseur a pour fonction de lui montrer ce qu'il n'a pas compris dans ce qu'il rapporte, notamment devant les autres dans le cas de supervisions dite collectives (c'est-à-dire des supervisions individuelles, se déroulant en groupe).

Les multiples manifestations possibles de transfert et de contre-transfert de part et d'autre ont donné lieu à de nombreux travaux, notamment à propos de l'analyse dite quatrième (IVième Groupe): l'analysant, l'analyste en supervision, son analyste et le superviseur (de Mijolla-Mellor, 2002; Valabrega, 1979). La supervision ne donnant lieu à aucune validation institutionnelle peut être considérée comme une forme d'analyse personnelle qui a des spécificités notamment par la pluralité des références. S’y associent pour l'habilitation les «sessions interanalytiques». Si l'analyste du superviseur était 
également pris en compte, on aurait l'analyse cinquième ouvrant la question de la transmission intergénérationnelle de la psychanalyse par les supervisions : affiliation et filiation...

Il est plus généralement admis que la perlaboration des implications transférentielles et contre-transférentielles fait partie de la supervision. Par exemple, la répétition dans le conflit avec le superviseur du conflit entre le patient et le supervisé peut être analysée, mais il arrive que le supervisé paraisse ressembler au patient dont il parle: il sélectionne inconsciemment ce qui lui ressemble chez son patient. S'il change de patient, ce qu'il en rapporte ressemble au patient précédent... La répétition à positions inversées peut constituer une tache aveugle. Ces phénomènes de réverbération requièrent ce que L. de Urtubey appelle le «travail de contre-transfert », ils peuvent rendre souhaitable le retour à l'analyse personnelle (parfois en cours) ou une nouvelle tranche...

\section{Une contradiction interne de la supervision}

La logique secondaire de mise en forme narrative est plus ou moins tributaire d'une logique discursive. Le récit des énoncés du patient tend à faire disparaître l'énonciation, l'intonation, les affects dans l'expression verbale et extra-verbale. D’où le lissage narratif, secondarisé, défensif (a fortiori obsessionnel), qui gomme les discontinuités, les silences. Le récit du supervisé, a fortiori en groupe, occulte nécessairement la pesée des processus primaires de l'inconscient pulsionnel, c'est-à-dire le contre-transfert au sens strict ainsi que le refoulement corrélatif, pesée sur la perception du matériel et sur les interprétations et les constructions.

En somme, la contradiction fondamentale peut être ainsi formulée: rendre compte pour l'écoute flottante du superviseur d'une écoute supposée flottante du supervisé, tout en enregistrant systématiquement le matériel pour en rendre compte clairement, d'où la tentation de prendre des notes pendant la séance - un symptôme qui demande analyse. Le désir de ne rien oublier et de maîtrise omnipotente est rapidement confronté aux effets du passage par l'écriture des notes. La réflexion qu'elle suppose inspire une autre compréhension du matériel et constitue une première auto-supervision propre à inspirer l'interprétation en identification à l'écoute anticipée du superviseur. C'est l'effet souhaitable du processus de formation mais, au moins au début, la réduction narrative secondarisée se manifeste dans les notes remises au superviseur. Et aussi, parfois, dans le rapport du superviseur : un récit de cas qui fait plus de place à la biographie compréhensive du patient qu’à 
l'analyse de l'inconscient et des transformations psychiques qui en ont résulté.

Une des implications de ces remarques est de savoir s'il faut ou non exclure dans la séance de supervision la lecture de notes (évidemment prises en dehors des séances, voire à distance de celles-ci). Les exigences des superviseurs sont diverses: l'exclusion imposée peut laisser place à des efforts de mémoire plus ou moins laborieux impliquant le refoulement des éléments les plus vifs du matériel et un appauvrissement considérable, notamment dans ce qui comporte transfert et contre-transfert. La performance mnésique fait illusion quand elle repose sur la relecture précipitée des notes dans l'ascenseur. Il faut dire que la mémorisation est plus ou moins facile selon les patients et selon l'avancement dans l'expérience de l'analyse supervisée: il devient possible tôt ou tard de s'en tenir à des notes succinctes ou pas de notes du tout.

Les notes sont toujours au moins implicitement adressées et elles sont toujours l'effet de transformations et de distorsions. Dans un premier temps, elles donnent à l'analyste en formation l'expérience de sa propre supervision par la prise de conscience de ce qui a pu d'abord lui échapper et, au mieux, l'expérience de la confrontation des données empiriques immédiates aux modèles théoriques, donc de l'articulation théorico-pratique dans l'activité de penser, de réfléchir (qui sera illustrée dans le futur mémoire de candidature au titulariat ainsi préparée). Dans le cadre de la supervision du cursus, elles impliquent le tiers observateur et interprétant sinon l'instance surmoïque qu'incarne le superviseur. On continue à parler de «contrôle» et de « contrôleur» (de l'existence duquel le patient peut avoir l'intuition en lui donnant figuration dans le matériel, comme l'avait noté F. Pasche) : l'interprétation décalée dans le temps laisse supposer l'intervention d'un tiers. L'enregistrement mnésique du matériel pour un autre tend à exclure la régrédience chez l'analyste, la relative régression topique que requiert l'écoute métapsychologique, laquelle rend possible la spontanéité interprétative, la créativité analytique...

\section{Les effets négatifs de la situation de supervision}

Le risque d'interruption de l'analyse par le départ du patient exerce souvent une contrainte sur l'indication, sur la technique et sur le contre-transfert. Elle induit à forcer l'indication, à ne pas prendre de risque interprétatif ou l'inverse (trop de précipitation), le surcroît d'intérêt et la hantise de l'échec (selon un questionnaire récent, un tiers des patients en analyse de supervision du cursus interrompent leur analyse au bout d'une durée très variable). La 
problématique de l'identification à l'analyste de l'analyse personnelle, souvent encore en cours, est toujours en jeu.

Par rapport à l'expérience souvent prolongée de la pratique des psychothérapies, une rupture avec les acquis et les routines est nécessaire et parfois douloureuse. Elle demande un apprentissage de la neutralité, de la passivité réceptrice, de la tolérance au doute, de la «capacité négative» (selon Bion): une élaboration qui peut durer longtemps et qui est tout à fait à l'opposé de la pratique médicale.

La co-associativité de l'analyste et de l'analysant, valorisée par ceux qui soulignent le continuum entre psychanalyse et psychothérapie, peut tendre à exclure l'effacement de l'analyste et la neutralité dans l'écoute en égal suspens, mais aussi l'interprétation proprement dite ou encore, celle-ci est limitée au registre préconscient.

La supervision, par l'enregistrement au moins mnésique des données qu'elle requiert, peut induire les mêmes défauts que ceux qui sont dans les automatismes des psychothérapies: le codage psychanalytique des contenus manifestes, de la biographie, et de l'anecdotique, en ignorant les manifestations de l'inconscient, la clinique du transfert et le processus. D'où par exemple, en dehors des erreurs grossières de la suggestion et du conseil, la demande faite au patient de précisions factuelles inutiles, des interventions pour clarifier sa propre pensée, pour réduire les contradictions (ou confronter le patient à ses contradictions) par intolérance à l'ambiguité pourtant significative. Au pire, la pente de la psychothérapie cognitive ou du coaching...

Quand la règle de l'analyse dans le transfert devient un système, le matériel est considéré comme toujours directement adressé à l'analyste. Il est alors réduit à sa valeur de communication dans la disparition de l'intrapsychique, du transfert comme répétition de l'infantile. Dans le même sens, l'extension large et floue de la notion de transfert et de contre-transfert (le «transférocontre-transférentiel » que Pontalis considère à juste titre comme typique de la langue de bois psychanalytique) joue comme légitimation de tout et n'importe quoi dans l'effacement des différences topiques, comme si le transfert au sens strict comme quiproquo anachronique, ne supposait pas le processus, la mobilisation de l'organisation psychique bien différente de la simple relation interpersonnelle, des attitudes et des contre-attitudes. À partir d'analogies, le matériel (le manifeste) peut ainsi être systématiquement interprété par l'analyste comme référé à lui-même, ce qui peut empêcher le transfert, ou du moins son expression, en le supposant installé et utilisable. Le narcissisme de l'analyste débutant l'induit à ne pas se laisser oublier comme interlocuteur. 
Exclu, il a tendance à agir. Il arrive qu'il soit frustré de son désir de continuer son analyse personnelle à rôles inversés (un critère négatif de l'admission au cursus pourrait être l'absence d'élaboration de la fin d'analyse, du deuil de l'analyse idéale).

Le désir d'être important et que l'analyse soit importante dans la vie du patient peut susciter le recours contre-transférentiel à la suggestion, à la prescription illusoire du transfert, alors qu'il s'agit par exemple de la résistance au transfert. Un autre effet est la centration exagérée sur soi aux dépens de l'écoute de l'analysant, donc l'utilisation de celui-ci pour la poursuite de sa propre analyse, jusqu'à perdre de vue durablement la différence entre les deux scènes. Il est vrai qu'un moment ainsi défini peut être bénéfique dans l'instauration du «tiers analytique » (Ogden), de la «chimère » (de M'Uzan), mais le surcroît d'implication requiert aussi la capacité de la désimplication nécessaire pour l'interprétation.

La conscience de l'existence permanente du transfert dans diverses dimensions permet de l'utiliser sans interpréter systématiquement ses occurrences directes. Strachey (1934) avait déjà montré (avec des métaphores militaires, il est vrai) que des interprétations extra-transférentielles peuvent préparer et rendre possible l'interprétation transférentielle dite "mutative». Elles peuvent être fondées sur le transfert tout en évitant une trop grande proximité effractive qui limiterait l'extension de ses effets analytiques. L'interprétation du transfert ou dans le transfert peut être un acting contretransférentiel ne serait-ce que parce qu'elle est faite à contretemps. Elle n'exclut pas la subtilité de sa mise en œuvre dans l'anticipation de ses effets sur le fonctionnement psychique du patient à ce moment-là, donc sur le processus. La réduction du clivage (par exemple dans l'accès à et dans l'élaboration de la position dépressive) n'a pas les mêmes effets que la levée du refoulement (par l'interprétation du fantasme inconscient et de l'œedipe).

\section{Supervision et échange interanalytique}

La pensée clinique se fonde sur un savoir susceptible de fonder la prévision, l'anticipation, savoir qui est le fruit de la culture et de l'expérience et fait la différence, l'asymétrie, entre supervisé et superviseur (laquelle redouble diversement la différence des sexes et des générations). La supervision ne saurait, sans déni, être réduite à l'écoute en second, à l'échange interanalytique tel qu'il est en œuvre entre les membres de l'institution psychanalytique.

La crainte d'inféodation du supervisé aux théories préférées de son superviseur peut être partagée par les deux au risque du clivage théorico-pra- 
tique. Celui-ci peut résulter aussi chez le supervisé de ses difficultés de compréhension de la théorie, de l'insuffisance de ses connaissances ou de leur caractère fermé, simplifié, arrêté (qui peut résulter de l'expérience des psychothérapies). En ce sens, l'oedipe, qui est évidemment un modèle fondamental de l'organisation psychique inconsciente, peut être utilisé comme un code a priori, formant un système d'interprétation toujours vrai ( «l'œedipianisation ») : c'est aussi le danger de l'utilisation inconsidérée du modèle des fantasmes originaires. Toujours vrais par principe, ils peuvent écarter d'autres modèles théoriques utiles: le prégénital, la dialectique de l'organisation narcissique avec l'objectalité, la position dépressive, la problématique limite, les modèles de la relation mère-enfant précoce, le transitionnel. Il reste que la question de leur rapport avec l'organisation œedipienne des désirs et des identifications ne cesse de se poser, notamment dans leur fonction défensive anté et anti-œdipienne.

Ne sont pas immédiatement accessibles aux débutants venus de l'expérience des psychothérapies, les effets analytiques de la régrédience dans le contre-transfert, de la "chimère», de l'utilisation du «tiers analytique » comme objet analytique spécifique, mais aussi de la notion bionienne de la "capacité de rêverie de la mère», ou encore la perspective transgénérationelle. Le risque est d'adopter de manière spéculative ce qui a été décrit dans des cas ou des moments particuliers dans certaines cures. La critique de «l'empathisme» par S. Bolognini (1997) n'est pas sans pertinence. Le risque est que des publications de l'ordre de la recherche jouent comme modèle immédiatement et toujours utilisables ou encore, que l'insistance sur le continuum psychanalyse-psychothérapie entrave la distinction entre les interventions de type psychothérapique qu'il faut savoir assumer et les interprétations spécifiquement psychanalytiques. Des modes d'intervention novateurs supposent une grande expérience de l'analyse classique dans des indications de cure classique, c'est bien ce que l'on peut attendre des supervisions du cursus d'analyses aussi "classiques" que possible: «le laboratoire central» (Pontalis, 2012). À partir de là, prend toute son importance l'éventuelle supervision d'une psychothérapie psychanalytique. Non obligatoire, elle est souvent faite pendant ou après le cursus.

\section{La pratique de la supervision}

Au minimum, elle peut être considérée comme simple évaluation de la capacité de l'analyste en formation de conduire une analyse et de la mener à bien même dans les moments critiques. Dans ce cas elle peut être très brève. 
Elle n'a pas l'ambition d'être une formation: l'expérience de la pratique et les séminaires sont censés suffire. En somme, elle prolonge la validation du cursus, elle confirme la décision de cursus clos et validé comme habilitation à la pratique spécifiquement psychanalytique. Par contre, si la supervision est considérée comme processus de formation par étapes (Dispaux, 2002), ce qui est souhaitable, elle doit être plus longue et d'une durée de deux ou trois ans au minimum.

Quatre dimensions schématiques de la supervision peuvent être distinguées. Elles peuvent être alternatives, ou successives comme des moments différents d'une progression pédagogique. Ces différences de pratique de la supervision ne sont évidemment pas sans rapport avec le style de la pratique psychanalytique habituelle du superviseur...

1. La supervision comme nouvelle analyse personnelle ou comme perlaboration de l'analyse personnelle est caractérisée par la centration sur le contre-transfert du supervisé et son transfert sur le superviseur (en identification ou non au patient). Une "analyse quatrième » (Valabrega, 1979) ou cinquième (celle du superviseur), individuelle et «collective», peut alors être envisagée. Transfert et contre-transfert sont ici entendus au sens propre de phénomènes inconscients. Si elles poursuivent ou prolongent l'analyse personnelle, les supervisions ne peuvent être qu'individuelles et successives comme à l'APF. Si le supervisé est encore en analyse, le superviseur est alors en double...

Selon C. Stein (1971) par exemple, le principe de la poursuite par le psychanalyste de son analyse avec celle de son patient est généralisé: «Les séances du patient ont les meilleures chances de déboucher sur sa psychanalyse si elles sont pour son psychanalyste le lieu privilégié de la poursuite de la sienne... » (p. 364). A fortiori en supervision, si $\mathrm{du}$ moins cette pratique a encore un sens.

2. La démonstration. Il s'agit de « décortiquer» tout le matériel dans le détail de manière systématique comme une démonstration faite au supervisé réduit à admirer et à imiter. Le risque est l'usage dogmatique de la métapsychologie («toujours», «absolument», etc.), l'imitation à défaut d'identification à la fonction, à la quête de vérité.

3. La directivité. Dire ce qu'il faut dire, ce qu'il faut faire, par exemple pour l'instauration du cadre: les dates de vacances, le paiement, mais aussi les interprétations qui doivent être faites. Parfois même, il y aura incitation du superviseur à fréquenter son séminaire, sinon à 
devenir son élève afin qu'il promeuve ses idées... Le narcissisme d'auteur, de théoricien, pousse à faire valoir ses idées : convertir des adeptes sinon avoir son "fan-club". Les supervisés sont induits à donner des interprétations pour faire plaisir au superviseur...

Le savoir du superviseur est généralement inducteur d'une position d'élève et facteur d'idéalisation, d'où la position de passivité et de soumission vis-à-vis du maître: facteur d'inhibition ou ressort dynamique? Le mieux est l'évolution de l'un à l'autre dans les temps différents du processus.

Les avantages et surtout les inconvénients de la relation maître-élève, maître-disciple, sont bien illustrés par l'échec de Lacan. Il a créé une École dans laquelle il exerçait un pouvoir absolu pour la promotion d'un système de pensée devenu dogmatique chez ses élèves, et sans trouver d'issue au problème de l'habilitation des analystes ( «'Lanalyste ne s'autorise que de lui-même»; il ajoute par la suite « et de quelques autres...»). F. Roustang (1976) en a fait une critique radicale en partant du destin de certains élèves directs de Freud.

4. À l'inverse, savoir ne pas savoir. Dans l'attention à ne pas priver le supervisé de la direction de la cure, le superviseur indique les mouvements, les moments significatifs, et s'en tient au repérage du transfert, des transferts, et du contre-transfert seulement s'il fait obstacle sans que l'analyste en formation le perçoive. Le but est le développement de la capacité contre-transférentielle, c'est-à-dire la constitution du cadre interne de l'analyste avec sa double face: perception du transfert et endo-perception du contre-transfert. S'y adjoignent les conseils, l'aide à la compréhension du cas, à ce que Bouvet appelait «l'élaboration intuitive du cas».

Mais aussi :

- proposer plusieurs hypothèses interprétatives, des alternatives: pour éviter que celle du superviseur soit reprise telle quelle à froid, décalée par rapport à l'évolution, perdant son efficace;

- ne pas répéter une critique, c'est-à-dire laisser faire les erreurs sans gravité, les interventions inutiles, sans les relever directement, mais montrer in situ la différence entre interventions psychothérapiques et interprétation analytique;

- laisser le choix à propos des problèmes de maintien du cadre et aider à en repérer les effets.

Dans tous les cas, l'essentiel est: 
- de montrer comment les interventions peuvent être entendues par le patient autrement, sur un autre plan que celui visé, ce qui peut être analysé par méta-communication;

- montrer les effets directs, indirects, immédiats et différés des interventions (plus repérables avec la mise en série des séances dans la supervision);

- la clarification et l'élucidation des modèles théoriques implicites, des interprétations latentes, et plus généralement du sens du vocabulaire freudien; l'illustration clinique des concepts a ici le plus grand rôle.

Par ailleurs, faut-il conseiller de lire en fonction du cas? C'est un moyen privilégié d'appropriation de la théorie, de relance de l'interrogation et de la réflexion. Le risque est celui de l'intellectualisation au service du refoulement, de la mise à distance de l'implication subjective, de l'écoute biaisée par l'intérêt personnel d'une lecture, donc de l'induction d'un matériel défensif, artificiel. Il peut en être ainsi quand le matériel est pauvre, dispersé, hétérogène, insaisissable et que, de ce fait, la supervision devient ennuyeuse. À mon avis, mieux vaut un temps différent pour la théorie afin d'éviter les effets de brouillage et de confusion, et surtout d'accréditer l'idée de transmission d'un savoir constitué jouant comme référence dogmatique, comme système de pensée fermé et comme doctrine, comme on disait autrefois.

\section{L'appropriation de la fonction analytique}

Il s'agit ici de l'appropriation, pas tant de la technique que de la fonction analytique, voire de l'identité et de l'éthique psychanalytique, de l'écart fonction-sujet décrit par J. L. Donnet (2009). L'appropriation de la fonction, qui est le but de la transmission de la psychanalyse, a des conditions difficiles à définir puisqu'elles échappent à tout vouloir et à tout pouvoir.

Le fait qu'il y ait toujours au moins deux supervisions avec deux superviseurs différents dont l'une en groupe (dite collective), limite a priori le risque d'inféodation, d'imitation simple, d'aliénation par le savoir. Les séminaires, les groupes de travail, la pratique du psychodrame individuel en groupe, et les échanges internationaux vont dans le même sens: celui de la pluralité des références, mais au risque de la dispersion, de l'incohérence et du relativisme. L'autonomisation de la pratique de l'analyste en formation (puis du jeune analyste) le rend apte à utiliser sa réalité psychique propre, tout en respectant le principe de la neutralité, laquelle suppose l'alternance d'implication et de désimplication subjective. 
Dans les questionnaires remplis par les analystes en formation (1996, 2001, 2009), les réponses les plus nombreuses ont toujours exprimé la satisfaction de l'ambiance conviviale des supervisions et, par là, le sentiment d'appartenir à une institution vivante.

Trois expériences personnelles du supervisé sont à considérer :

- celle de l'humour qui a sa place dans la supervision. J. L. Donnet a récemment décrit son effet de modification des rapports moi-surmoi;

- celle de petites transgressions par rapport à l'idée que se fait le supervisé de l'orthodoxie;

- celle de la découverte des limites du superviseur et de l'analysabilité elle-même. Le superviseur s'avère contestable, parfois confiné dans ses préférences techniques et théoriques. Ses prévisions ne sont pas toujours confirmées, et en un mot, il est avéré qu'il n'est pas omnipotent et omniscient.

Lors des supervisions collectives, chacun est en tiers observateur de la supervision d'un autre, et réciproquement. Cette formule originale a donné lieu à peu de commentaires. Néanmoins, celui de Serge Viderman (1980) est très critique: déjà le mot «supervision » en français est jugé trop normatif, le patient en ferait les frais parce que son analyste serait inhibé par la contrainte de devoir rendre compte du matériel devant le superviseur et devant les autres, ce qui implique notamment une transgression de la règle de confidentialité. De ce point de vue, faut-il exclure l'analyse d'un patient psychiatre ou psychologue?

Le commentaire de Jean Guillaumin, plus récent (ou moins ancien, puisqu'il date de 1999), est au contraire très enthousiaste : il y voit l'enrichissement de l'expérience analytique en identification imaginaire aux autres supervisés, et une excellente préparation à la vie institutionnelle et aux échanges entre analystes. Cependant, pour Guillaumin, il s'agit aussi d'une sorte d'analyse de groupe. Ainsi, il établit une règle de restitution de tout ce qui se dit entre les supervisés en dehors de la séance.

Il arrive que les difficultés d'un supervisé en supervision collective donnent lieu à une élaboration dans la supervision individuelle. Tel est le cas du supervisé qui arrive devant un groupe constitué depuis longtemps, ayant pour règle l'interdiction d'utiliser les notes: les effets sur l'analyse sont préjudiciables quand l'impératif surmoïque écrase la liberté associative et l'initiative interprétative... d'autant plus que la situation de groupe peut renforcer l'idéalisation du superviseur. Il est important que se trouvent mises en cause d'une manière ou d'une autre les projections surmoïques et 
l'idéalisation, sinon la sacralisation du superviseur. L'élaboration de la pratique psychanalytique requiert la capacité de mise en cause des idées reçues, et d'une lecture critique de Freud comme ouvre ouverte, dans son pouvoir génératif renouvelé par de nouvelles lectures.

La perception des différences entre les règles fondamentales de la méthode et les normes de groupe, l'idéal institutionnel, et les usages (les mimétismes, les automatismes interprétatifs, les routines), conjure le risque de faux-self psychanalytique. L'exploration tâtonnante d'un style personnel permet l'élaboration de l'identification transférentielle à l'analyste de l'analyse personnelle plus ou moins transférée sur le superviseur. Le transfert du transfert devient idéalement «transfert de travail », mais cette notion fort ambiguë n'est peut-être qu'un reste de l'analyse didactique ou espérée didactique?

Les conditions du développement de la créativité analytique et du style personnel ne sont pas sans rapport avec la nature transgressive de l'aventure analytique... Elles préparent la fin de la supervision, donc le travail de la séparation pour l'analyste en formation et pour le superviseur. Du reste, le supervisé peut toujours revenir donner des nouvelles de la suite de la cure, ce qui a un intérêt tout particulier en supervision collective.

\section{Pour conclure}

La supervision de la pratique analytique du débutant dans une cure aussi «classique » que possible est toujours un élément fondamental du cursus de formation. Elle a plusieurs objectifs :

- L'évaluation de la capacité de la fonction. Celle-ci demande une certaine durée qui dépend de l'évolution de la cure supervisée. Bien que les superviseurs puissent s'en faire assez vite une certaine idée, elle ne peut être établie qu'au terme des deux supervisions lors des réunions de fin de cursus. Ainsi le superviseur peut-il utilement l'oublier en cours de supervision.

- Une expérience fondatrice. Notamment au début, elle comporte une certaine reprise élaborative de l'analyse personnelle à rôles inversés. Elle a pour but la constitution du cadre interne de l'analyste par l'appropriation de la fonction.

- Une formation. L'art du superviseur est de faciliter, sans la diriger luimême, la cure, et ce, sans induire une relation analyste-analysant ni une relation maître-élève inductrice de passivité et de soumission, tout en conduisant un processus de formation. Ce faisant, le superviseur promeut le développement d'un style personnel de pratique analytique 
ordonné à la finalité fondamentale de la psychanalyse : celle de la quête de vérité.

Comme la psychanalyse, sa transmission - dont la supervision - est certes, un métier impossible... au regard de l'idéal.

Bernard Brusset 40 avenue d'Italie

Paris 75013

bernard.brusset@wanadoo.fr

\section{Références}

BOLOGNINI, S., 1997, Empathy and «empathism », International Journal of Psychoanalysis, 78 (2), 279-293.

BOLOGNINI, S., 2006, L'empathie psychanalytique, Ramonville Sainte-Agne, Érès.

BRUSSET, B., 2011, L'éthique dans la formation des psychanalystes, in B. Chervet et J. M. Porte (dir.), L'éthique psychanalytique, Paris, PUF, 145-160.

DISPAUX, M. F., 2002, Au cœur du processus de formation psychanalytique : transformation et transmission, in C. Botella (dir.), Penser les limites, Paris, PUF, 463-468.

DONNET, J. L., 2009, L'humour et la honte, Paris, PUF.

GUILLAUMIN, J., 1999, Quelques remarques sur les supervisions collectives, Revue française de psychanalyse, 63 (3), 943-957.

LEBOVICI, S., SOLNIT, A. J. (dir.), 1982, La formation du psychanalyste, Paris, PUF.

MIJOLLA-MELLOR (de), S., 2002, L'analyse quatrième, in A. de Mijolla (dir.), Dictionnaire international de la psychanalyse, Paris, Calmann-Levy.

PONTALIS, J. B., 2012, Le laboratoire central, Paris, Éditions de l'Olivier.

ROUSTANG, F., 1976, Un destin si funeste, Paris, Minuit.

STRACHEY, J., 1934, The nature of the therapeutic action of psycho-analysis. International Journal of Psychoanalysis, 15 (2-3) ; 1970, La nature de l'action thérapeutique de la psychanalyse, Revue française de psychanalyse, 34 (2), 255-284.

STEIN, C., 1971, L'enfant imaginaire, Paris, Denoël.

VALABREGA., J. P., 1969, Les voies de la formation psychanalytique, Topique, $\mathrm{n}^{\circ} 1$.

VALABREGA, J. P., 1979, La formation du psychanalyste. Esquisse d'une théorie, Paris, Pierre Belfond.

VIDERMAN, S., 1980, La machine dé-formatrice, Cahiers Confrontation, $\mathrm{n}^{\circ} 3$. 